\title{
Developing a chip-scale optical clock
}

\author{
Weimin Zhou, ${ }^{\text {a,* James Cahill, }}$, Jimmy H. Ni, ${ }^{\text {a }}$ Andrew Deloach, ${ }^{\text {a }}$ \\ Sang-Yeon Cho $\odot,{ }^{\text {a }}$ Stephen Anderson, ${ }^{\text {a,b }}$ Tanvir Mahmood $\odot$, \\ Patrick Sykes, ${ }^{\text {a,c }}$ Wendy L. Sarney, ${ }^{a}$ and Asher C. Leff ${ }^{a}$ \\ ${ }^{a}$ US Army CCDC-Army Research Laboratory, Adelphi, Maryland, United States \\ ${ }^{\mathrm{b}}$ Rensselaer Polytechnic Institute, Troy, New York, United States \\ ${ }^{c}$ University of Maryland, Baltimore County, Baltimore, Maryland, United States
}

\begin{abstract}
We report our in-house R\&D efforts of designing and developing key integrated photonic devices and technologies for a chip-scale optical oscillator and/or clock. This would provide precision sources to RF-photonic systems. It could also be the basic building block for a photonic technology to provide positioning, navigation, and timing as well as $5 \mathrm{G}$ networks. Recently, optical frequency comb (OFC)-based timing systems have been demonstrated for ultra-precision time transfer. Our goal is to develop a semiconductor-based, integrated photonic chip to reduce the size, weight, and power consumption, and cost of these systems. Our approach is to use a self-referenced interferometric locking circuit to provide short-term stabilization to a micro-resonator-based OFC. For long-term stabilization, we use an epsilon-near-zero (ENZ) metamaterial to design an environment-insensitive cavity/resonator, thereby enabling a chipscale optical long-holdover clock. (C) The Authors. Published by SPIE under a Creative Commons Attribution 4.0 Unported License. Distribution or reproduction of this work in whole or in part requires full attribution of the original publication, including its DOI. [DOI: 10.1117/1.OE.60.2.027107]
\end{abstract}

Keywords: optical clock; integrated photonics; optical frequency comb; epsilon near zero; metamaterial; micro-resonator; oscillators; chip-scale; environment-insensitive; time transfer; positioning; navigation and timing.

Paper 20201271 received Oct. 26, 2020; accepted for publication Jan. 29, 2021; published online Feb. 27, 2021.

\section{Introduction}

A wide range of scientific and commercial systems require time synchronization with increasingly high precision and accuracy, including very long baseline interferometry (VLBI), particle colliders, global navigation satellite systems, and 5G communications networks. Such time synchronization is typically achieved through an external communication network, such as the internet, dedicated satellite links, or free-space optical time transfer. ${ }^{1,2}$ Holdover clocks can reduce the required update rate of time synchronization signals for such systems and also allow operation in the event that these synchronization signals are temporarily unavailable, for example during a network outage. Many of these same applications, such as VLBI at microwave frequencies, would benefit from a frequency reference with not just long-term frequency stability, but also with low phase noise - or equivalently short-term frequency stability.

Most holdover clocks are based on architectures for probing atomic transitions, which enable good long-term frequency stability. However, the physics package containing the atoms to be probed as well as environmental shielding contribute to an overall size and cost that limits the systems' size, weight, and power consumption, and cost (SWaP-C). The chip-scale atomic clock (CSAC) ${ }^{3}$ which is commercially available from vendors such as Microchip and Teledyne, is a state-of-the-art example of a miniaturized atomic clock, and indeed the size, and cost of a CSAC is much lower than other atomic clocks. Nevertheless, the required vapor cells are not fully integrated, adding to the size, and the environmental shielding adds cost.

Furthermore, the vast majority of holdover clocks operate at relatively low frequencies (e.g., less than $10 \mathrm{GHz}$ ). In this case, low-phase-noise operation can be achieved straightforwardly by

*Address all correspondence to Weimin Zhou,weimin.zhou.civ@mail.mil 
the addition of a quartz oscillator in the loop. However, for holdover clocks that operate at frequencies at or above $10 \mathrm{GHz}$, low-phase-noise operation is more challenging to achieve, as low-phase-noise references at frequencies above $10 \mathrm{GHz}$ are not widely available.

In this paper, we propose a chip-scale optical holdover clock (CSOC) that would use a CMOS-compatible epsilon-near-zero (ENZ) optical resonator to provide a laser with enhanced long-term frequency stability, an optical frequency comb (OFC) to divide the stabilized optical frequency to a microwave, and a CMOS-compatible delay-line-based stabilization scheme to reduce the phase noise of the generated microwave. The all-CMOS-compatible architecture of the proposed clock may allow it to have SWaP-C that is even lower than that of a miniaturized atomic clock, such as the CSAC. Such a reduction in SWaP-C would allow the CSOC to be used in a wider array of circumstances. The remainder of the paper is structured as follows. In Sec. 2, we discuss the overall architecture of the proposed CSOC and the design philosophy. In Sec. 3, we discuss our experimental progress toward achieving a short-path-delay-line-based selfstabilization system that would allow the proposed clock to generate a microwave signal with low phase noise. The architecture was designed such that it could also be realized on a photonic integrated chip (PIC) in a CMOS-compatible process. In Sec. 4, we discuss our experimental progress toward achieving the environmentally insensitive ENZ resonator that will grant the CSOC its long-term frequency stability. This includes developing wavelength thick ENZ indium-tin-oxide (ITO) metamaterial and fabrication of air-core in ENZ ITO resonator devices.

\section{Design Concept for CSOC}

The principal objective of our design effort is to develop an optical holdover clock that generates a microwave signal with good frequency stability and low phase noise while minimizing its SWaP-C. The current state-of-the-art optical clock consists of three major components: ${ }^{4-8}$ (1) an ultra-low-expansion (ULE) cavity stabilized laser that generates an optical frequency with ultra-narrow linewidth (low phase noise), (2) an atomic reference that disciplines the ULE-cavity-stabilized laser in order to provide it with long-term frequency stability while preserving its low phase noise, and (3) an octave-spanning OFC that coherently divides the low-phase-noise and long-term-stable optical frequency down to a microwave frequency. Our approach to achieve a CSOC is to develop an on-chip replacements for each of these three laboratory components while maintaining the same purpose for each of these components. The goal is to eliminate the high SWaP-C ULE locked laser and the atomic reference. With our new innovative design concept, our proposed CSOC is made by a micro-resonator-based OFC that is simultaneously stabilized with an on-chip delay-line interferometer and an environmentally insensitive ENZ resonator.

\subsection{Concept of Micro-Resonator OFC for Frequency Division}

An immediate choice for achieving a reduction in size is to replace the traditional mode-lockedlaser-based OFC with a micro-resonator-based OFC. ${ }^{9-13}$ Indeed, other groups are developing octave-spanning micro-resonator-based $\mathrm{OFCs}^{14,15}$ including as part of at least one effort to develop an entire on-chip optical atomic clock. ${ }^{14}$ Hence, in the remainder of this paper, we will not focus on the development of the micro-resonator-based OFC.

\subsection{Concept for Achieving Short-Term Stability}

The ULE cavities that provide optical clocks with exceedingly low phase noise are typically $10 \mathrm{~s}$ of $\mathrm{cms}$ long ${ }^{16}$ and typically require high-SWaP-C external housing to provide environmental isolation. Here, we propose to replace the ULE-cavity-stabilized laser with a photonic-integrated short-path delay-line interferometer that stabilizes the OFC without the need for an intermediate laser. ${ }^{17-19}$ Although optical-fiber-based delay-line interferometers are a well-established tool to stabilize single-frequency lasers, ${ }^{17}$ they are not commonly used on PICs. By bringing this technique to the PIC platform, we face a major challenge to overcome the waveguide propagation loss, which is orders of magnitude higher than the propagation loss of optical fiber. 
Buried waveguides on the common 220-nm silicon-on-insulator platform typically achieve propagation losses of approximately $2 \mathrm{~dB} / \mathrm{cm}$ at wavelengths near $1550 \mathrm{~nm} \cdot{ }^{20}$ By moving to more complicated waveguide geometries, silicon waveguides have been reported with losses below $3 \mathrm{~dB} / \mathrm{m}^{21-23}$ Additionally, some approaches toward ultra-low-loss $\mathrm{Si}_{3} \mathrm{~N}_{4}$ waveguides have been reported demonstrating less than $1 \mathrm{~dB} / \mathrm{m}$ loss. ${ }^{24,25}$ Nevertheless, regardless of the technology, the propagation loss of an integrated photonic delay line is significantly higher than the $\sim 0.17 \mathrm{~dB} / \mathrm{km}$ propagation loss of commercial optical fiber.

Our expectation of increased propagation loss of the integrated photonic delay line had a significant impact on the design of our interferometer. First, we limited the interferometer delay to less than $10 \mathrm{~m}$, which significantly decreased the sensitivity of the interferometer. Second, even with a relatively short delay, we expect a high power imbalance between the delayed and non-delayed components of the light at the output of the interferometer, which may result in an increased contribution from laser relative intensity noise. Consequently, our development strategy was to first construct a fiber-optic prototype to demonstrate that a short-path interferometer has sufficient sensitivity to enable the generation of a low-phase-noise microwave. Future work will extend this prototype to an integrated photonic chip that stabilizes a micro-resonator-based OFC. Toward that end, we also designed and fabricated an integrated-photonic tunable coupler in order to mitigate the expected power imbalance. The results of these experiments are presented in Sec. 3.

\subsection{Concept of Environment-Insensitive Device for Long-Term Stability}

Any oscillator is susceptible to long-term frequency drift. One of the most pervasive sources of this drift arises from the oscillator's sensitivity to environmental fluctuations, including acoustic, vibrational, and temperature perturbations. The atomic reference in the laboratory-scale clock serves to mitigate this drift, because the atomic transition is relatively insensitive to the environment. Cold atoms are a particularly high-performing reference since they are extremely well isolated from the environment. Nevertheless, atomic sources inherently increase the SWaP-C of a clock.

Rather than pursuing an on-chip atomic reference, we propose an approach to obtain longterm frequency stability. We are developing an environmentally insensitive hollow-core microresonator that is clad with an ENZ metamaterial. ${ }^{26}$ Although we do not expect the frequency accuracy or frequency stability at very long timescales (e.g., $>10^{3} \mathrm{~s}$ ) of the ENZ reference to be competitive with cold atom references, we expect its frequency stability to compete with miniaturized atomic clocks such as the CSAC. Moreover, when we consider the possibility to incorporate the fabrication of the ENZ resonator into a CMOS process, we expect that the overall SWaP-C will be greatly reduced, resulting in a cheap, compact, and easily manufacturable frequency reference.

Our concept is to use the ENZ metamaterial as waveguide cladding to form an air or vacuum core resonator. ${ }^{26}$ Figure 1 shows a schematic illustration of the air-ring, ENZ-metamaterialcladding resonator device. The idea is to make an intrinsic environment-insensitive cavity/ resonator device.

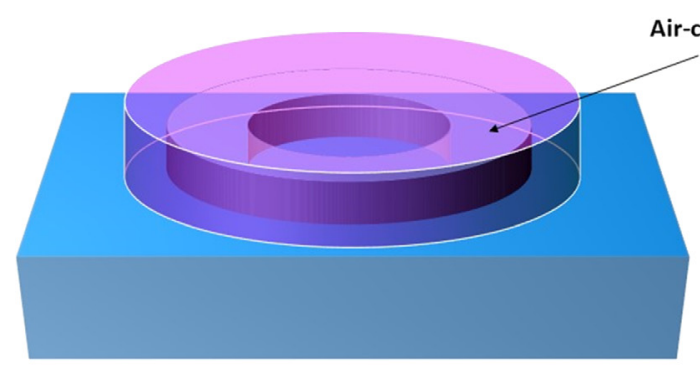

(a)

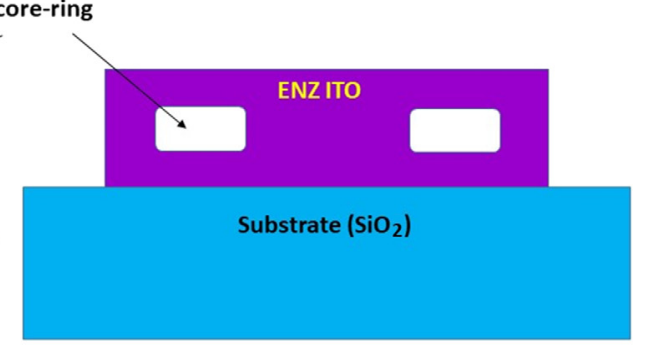

(b)

Fig. 1 Air-core-ring and ENZ-metamaterial cladding resonator: (a) 3D illustration and (b) crosssection view. 
For a typical ring resonator, the resonant optical frequency is $f_{\text {op }}=m \times f_{0}$, where $m$ is the number of modes and $f_{0}$ is the fundamental frequency. The fundamental wavelength $\lambda_{0}=C / f_{0}$, which is the round-trip optical path. Also, $\lambda_{0}=n \times d$, where $n$ is the effective refractive index of the ring path and $d$ is the physical dimension of the resonator. Both temperature and vibration may change the physical dimension of the resonator. In general, the environmentally induced frequency change is given by Eq. (1):

$$
\Delta f_{\text {op }}=m \times \Delta f_{0}=-m \times\left(\frac{\Delta n}{n}+\frac{\Delta d}{d}\right) f_{0} \text {. }
$$

In a typical micro-resonator containing $\mathrm{SiO}_{2}, \mathrm{Si}_{3} \mathrm{~N}_{4}, \mathrm{Si}$, etc., the change in refractive index, $\Delta n$, can be significant due to temperature changes and vibration-induced strains. In case of a material with thermal expansion $\alpha_{\text {th }}$, Eq. (1) can be written as Eq. (2):

$$
\frac{\Delta f_{\mathrm{op}}}{f_{0}} \cong-\alpha_{\mathrm{th}} \Delta T-\left(\frac{1}{n_{0}}\right) \times\left(\frac{d n}{d T}\right) \Delta T,
$$

where $\alpha_{\text {th }}$ is the thermal coefficient. The change in resonator physical dimension, $\Delta d$, can be significant due to thermal expansion $\left(\cong \alpha_{\mathrm{th}} \Delta T\right)$ and deformation by vibration.

In contrast, in a vacuum/air resonator embedded in ENZ metamaterial (e.g., in ENZ cladding layer), as provided by the embodiments herein, $\Delta n$ is negligible. The $\Delta d$ can be less significant due to the geometry of the material where vacuum is enclosed by the ENZ cladding layer. In addition, with the ENZ condition, optical phase changes very slowly in the ENZ media, thus environmentally induced small geometry variations have less effect on optical phase variation, and therefore generates less phase noise.

\section{Self-Referencing-Stabilization Locking of an OFC}

In this section, we review our experimental efforts toward realizing an integrated-photonic delayline interferometer that can stabilize an OFC. We constructed a prototype 8-m fiber-optic interferometer that stabilized a commercially available OFC in order to demonstrate that a short-path interferometer had sufficient sensitivity to significantly reduce the free-running phase noise of the OFC, thereby enabling low-phase-noise microwave generation. We found that the phase noise of the tone was reduced by over $35 \mathrm{~dB}$ when the OFC was stabilized with the short-path interferometer, reaching $-130 \mathrm{dBc} / \mathrm{Hz}$ at a $1-\mathrm{kHz}$ offset from the $10-\mathrm{GHz}$ carrier frequency. We also designed and tested tunable couplers that were fabricated by a commercial foundry (AIM Photonics). These tunable couplers will be used in future work to ensure that the delayed and non-delayed light components in an integrated-photonic interferometer have similar power.

\subsection{Proof of Concept Demonstration Using Fiber Delay-Line Interferometer}

The theory of operation of the delay-line-interferometer-stabilized OFC is as follows. The OFC illuminates a fast photodiode to generate a microwave with a frequency, $f_{m}$, that is an integer multiple, $m$, of the OFC's repetition rate, $f_{r}$, such that $f_{m}=m f_{r}$. Meanwhile, the optical power at the output of the interferometer, $d P(t)$, is proportional to fluctuations of the OFC's carrier frequency, $d f_{c}(t)$, according to $d P(t) \sim \tau_{g} d f_{c}(t)$, where $\tau_{g}$ is the group delay imposed by the interferometer delay. Notably, the carrier frequency of the OFC is nearly an integer multiple, $N$, of the repetition rate; it is given by $f_{c}=N f_{r}+f_{\mathrm{CEO}}$, where $f_{\mathrm{CEO}}$ is the carrier-envelope offset frequency of the OFC. If $f_{\mathrm{CEO}}$ is stabilized so that its noise contribution is negligible, then the output power of the interferometer is proportional to the noise of the repetition rate, $d f_{r}(t)$, according to $d P(t) \sim N \tau_{g} d f_{r}(t)$. This signal is filtered and fed back to the OFC in order to stabilize the repetition rate. The key point is that the interferometer's sensitivity to the repetition rate noise is proportional both to the length of the interferometer delay and to the integer $N$, which corresponds approximately to the ratio of the carrier frequency to the OFC's repetition rate and is typically on the order of $10^{5}$ or $10^{6}$. 


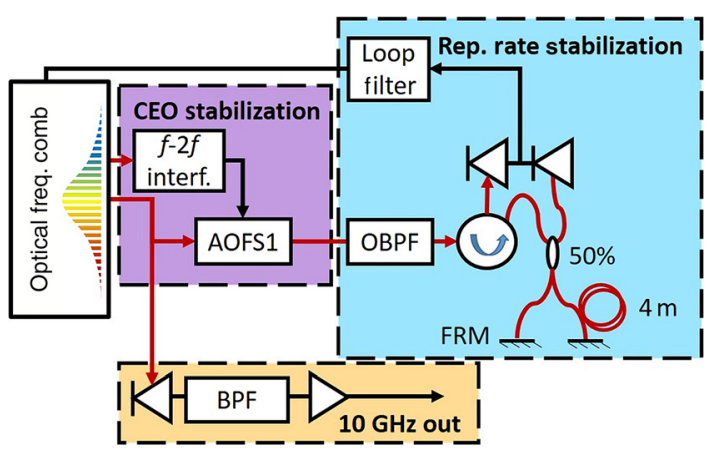

Fig. 2 System architecture used to stabilize the OFC. The $f_{\text {CEO }}$ is stabilized using an AOFS1 and the $f_{\text {rep }}$ is stabilized by a Michelson interferometer. A $10-\mathrm{GHz}$ microwave signal is generated by a photodiode.

One consequence of the higher propagation loss on an integrated chip is that the path imbalance in the interferometer will be limited to the scale of meters or less. This limitation is also related to the limited area available on a PIC. By contrast, the delay lines used to stabilize single-frequency lasers are typically kilometers long since the additional length increases the interferometer sensitivity. ${ }^{17}$ Hence, the integrated-photonic interferometer will necessarily have orders of magnitude lower sensitivity than the typical fiber-optic interferometer. Nevertheless, we expect that the fact that the interferometer is sensitive to frequency noise at the carrier frequency - and therefore proportional to the large integer $N$-will afford us some leeway in the sensitivity. To prove our concept for a short-path interferometer, we constructed a fiberoptic prototype interferometer with a 40-ns delay line, corresponding to $8 \mathrm{~m}$ of optical fiber. We stabilized a commercially available OFC with this interferometer and generated a $10-\mathrm{GHz}$ microwave signal with phase noise of $-130 \mathrm{dBc} / \mathrm{Hz}$ at $1-\mathrm{kHz}$ offset frequency. ${ }^{18}$

The architecture of the system used to generate the $10-\mathrm{GHz}$ signal is shown in Fig. 2. The OFC was a commercially available fiber-based comb from Menlo Systems with a center wavelength of approximately $1550 \mathrm{~nm}$ and a $f_{\text {rep }}$ of $250 \mathrm{MHz}$. A commercially available $f-2 f$ interferometer (Menlo Systems) measured the $f_{\text {CEO }}$ of the comb. The output of the OFC was split into two parts.

The first portion of the OFC output was used to generate the $10-\mathrm{GHz}$ signal. The light from the OFC entered a fast PIN photodetector (Optilab PD-20), where it generated a microwave frequency comb, with frequency components at the $f_{\text {rep }}$ of $250 \mathrm{MHz}$ and its integer harmonics. We used a microwave bandpass filter (BPF) with a center frequency of $10 \mathrm{GHz}$ to isolate the 40th harmonic and amplified it with a low-phase-noise RF amplifier. Since the frequency of the microwave output was directly related to the OFC repetition rate, its phase noise was also directly proportional to the phase noise of the OFC repetition rate.

The second portion of the OFC output was used to stabilize the OFC's repetition rate, thereby reducing the phase noise of the $10-\mathrm{GHz}$ microwave. The light from the OFC passed through an acousto-optic frequency shifter (AOFS1), which imparted a frequency shift on the OFC according to the frequency of the signal at its voltage input. This signal was the $f_{\text {CEO }}$ of the OFC and was provided by the output of the $f-2 f$ interferometer. The polarity of the frequency shift imparted by AOFS 1 was chosen such that it canceled the $f_{\mathrm{CEO}}$. The $f_{\mathrm{CEO}}$-free comb light entered a 0.8-nm optical BPF and then a fiber-optic Michelson interferometer with a path imbalance of 40 ns. We used single-mode fibers and non-polarization-maintaining components. The Faraday rotating mirrors mitigated the impact of polarization fluctuations. The output of the Michelson interferometer illuminated a set of balanced photodiodes, which converted the fluctuations of the $f_{\text {rep }}$ into a photocurrent. We used balanced photodiodes to reduce the current associated with the laser intensity noise. The photocurrent from the balanced photodiodes passed through a loop filter and fed back to an electro-optic modulator within the OFC cavity in order to stabilize the $f_{\text {rep }}$.

The phase noise of the $10-\mathrm{GHz}$ microwave generated by the OFC is shown in Fig. 3. When the OFC was free-running (i.e., when the self-stabilization circuit is turned off), the microwave 


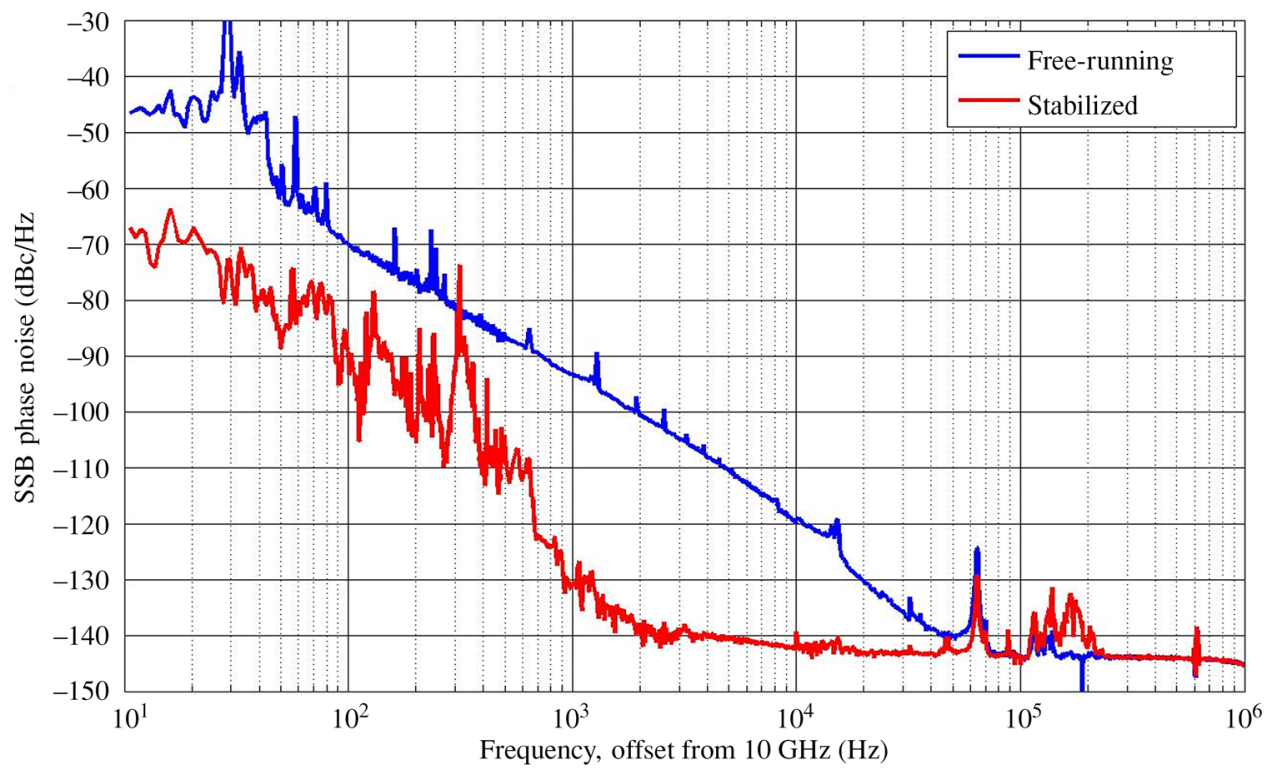

Fig. 3 Single sideband (SSB) phase noise of the 10-GHz microwave generated by the OFC when free-running (top, blue curve) and when stabilized (bottom, red curve).

had a phase noise of approximately $-93 \mathrm{dBc} / \mathrm{Hz}$ at a $1-\mathrm{kHz}$ offset frequency. When the OFC was stabilized, the microwave had a phase noise of approximately $-130 \mathrm{dBc} / \mathrm{Hz}$ at a $1-\mathrm{kHz}$ offset frequency. Hence, the self-stabilization circuit reduced the phase noise of the microwave by over $35 \mathrm{~dB}$ in this offset frequency range. In the stabilized case, the noise at offset frequencies below $1 \mathrm{kHz}$ is consistent with environmental fluctuations in the laboratory, such as temperature variations, vibrations, and acoustics. Hence, we believe that with improved packaging, the noise in this offset-frequency range will decrease.

These results demonstrate that a 40-ns fiber-optic interferometer has sufficient sensitivity to stabilize an OFC such that we could generate a microwave signal with ultra-low phase noise. We expect the interferometer on the integrated platform to introduce additional loss, so further work may be required to achieve similar performance with a PIC. On the other hand, due to its smaller size, an integrated circuit can be more easily isolated from environmental fluctuations than the fiber-optic prototype, which suggests that the noise at some offset frequencies may decrease. In any case, these results are a significant and encouraging step toward the realization of an on-chip stabilization circuit.

\subsection{Development of Integrated-Photonic Tunable Couplers}

Another consequence of the high propagation losses in a photonic integrated waveguide is that the loss imparted by a centimeters- or meters-long delay line imparts a large power imbalance between the interferometer paths. Such an imbalance may lead to exaggerated noise contributions from laser intensity noise. So, we designed, fabricated, and tested a 1-by-2 splitter with a tunable splitting ratio to balance the optical power of the short delay with a meter-long delay at the output combining point of the asymmetric interferometer.

The tunable 1-by-2 splitter was fabricated using a commercial integrated-photonics foundry (AIM-Photonics). We also designed and had fabricated a delay-line waveguide, an asymmetric Mach-Zehnder interferometer, and balanced photodetectors using the same process. Optical images of these components are depicted in Fig. 4.

The splitting ratio of the 1-by-2 power splitter is adjusted using a doped silicon heater that runs parallel to the evanescent coupling region. The silicon heater is partially etched to prevent mode coupling from the evanescent coupling region into the heater itself, which would otherwise cause excess loss. The thermal tuning performance is depicted in Fig. 5. This adjustable power split allows the unequal loss in the two interferometer paths to be offset so that the output power is roughly equal. The relative splitting ratio can be adjusted by up to $-35 \mathrm{~dB}$. 


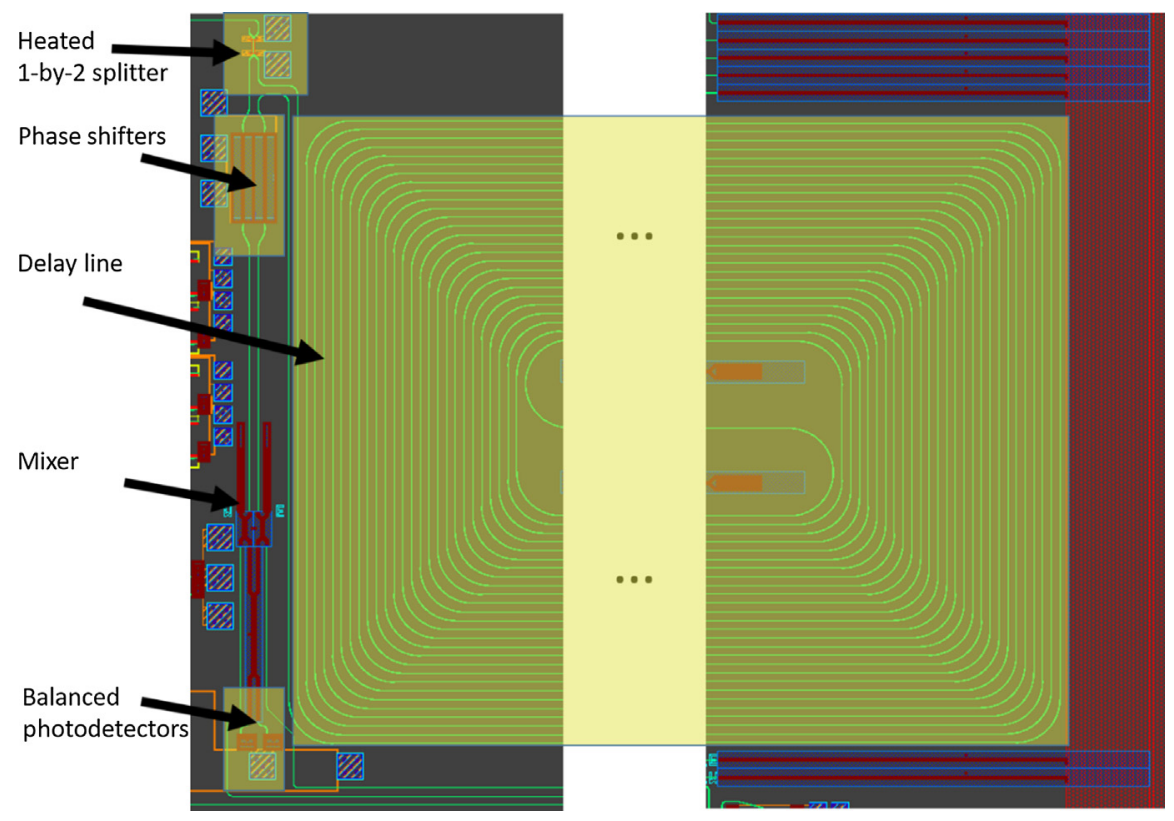

Fig. 4 Layout of the integrated lock circuit.

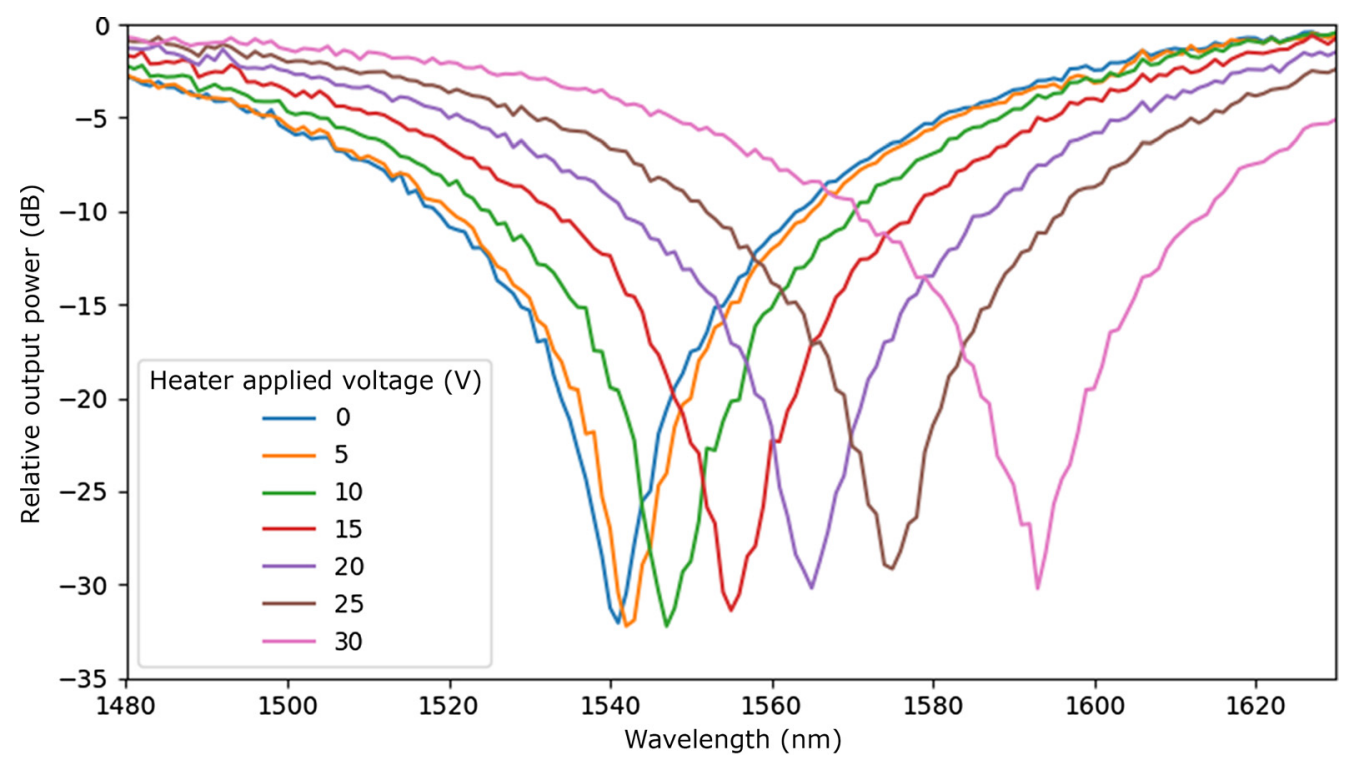

Fig. 5 Output power into one arm of the 1-by-2 power splitter, adjusted using a doped silicon heater. This adjustment of input power into the two asymmetric MZI arms can balance the net loss in each arm so that the output power in each path is roughly equal.

\section{Developing ENZ-ITO Metamaterial Based Environment-Insensitive Devices}

We outline our effort of developing the air-core, ENZ-metamaterial cladding resonator as environment-insensitive cavity device. First, we need to develop a suitable ENZ metamaterial for such a device. Although there are many reported ENZ metamaterials such as multi-layer metal-dielectric super lattice ${ }^{27}$ and nano-artificial metastructures, ${ }^{28}$ those metamaterials involve high-cost fabrication and may not be compatible with Si-photonics and CMOS processing. We chose ITO that is a practical thin-film material widely used in semiconductor optoelectronic devices. ITO has shown particular promise because of its potential for low-cost fabrication and 
compatibility with Si-based integrated photonics. However, in all the previously reported ENZ ITO film studies, the film thicknesses were significantly less than the wavelength region where ENZ properties occur. In our air-core, ENZ-metamaterial cladding resonator design, ENZ-ITO film needs to be around one wavelength thick. Since we would like to work at the telecom wavelength $(1.55 \mu \mathrm{m})$ in order to benefit from the majority of telecom photonic devices, we need to develop thick ENZ ITO metafilms.

\subsection{Thick ENZ-ITO Meta-Film Development}

We fabricated a variety $\sim 2-\mu$ m-thick ENZ ITO films via pulsed DC sputtering onto a $\mathrm{SiO}_{2}$ layer placed on top of a Si substrate and subsequently thermally annealed them. We performed a systematic study of these metafilms and discovered a non-uniform variation of the ENZ property. ${ }^{29}$

The experimental results of these ENZ properties are shown in Fig. 6. Since no equipment exists that can directly measure an unknown variable (vertically) permittivity, $\varepsilon$, of a multi-layer material system, we developed a comprehensive method to obtain the permittivity as a function of vertical location in the film. The method involves taking variable angle spectroscopic ellipsometry and fitting the measured data with an appropriate physical model, which also sliced the thick ITO film region into 30 thin layers.

The different zero-crossing frequencies are shown in Fig. 6(a), for 10 min annealing with temperatures ranging from $250^{\circ} \mathrm{C}$ to $400^{\circ} \mathrm{C}$. Figure $6(\mathrm{~b})$ shows that for all annealing temperatures, the ITO layer permittivity was not constant with respect to the depth. At lower annealing temperatures, the samples' permittivity depth profile showed a relatively small amount of non-uniformity, which is qualitatively consistent with the behavior reported for thin ITO films as shown in Fig. 6(c). However, at higher annealing temperatures, a sharp transition region appeared midway through the depth of the ITO film. Above this transition region-i.e., near the top of the ITO film - the real part of the permittivity was positive, whereas below the transition region-i.e., near the bottom of the ITO film - the real part of the permittivity was negative.

To understand this variation behavior, we conducted several microanalysis experiments on three of our ITO samples: as-deposited, annealed at $250^{\circ} \mathrm{C}$, and annealed at $350^{\circ} \mathrm{C}{ }^{29}$ Specifically, we examined the crystallinity and texture using x-ray diffraction (XRD), the crystallite morphology using cross-sectional transmission electron microscopy (TEM), and the chemical composition using energy dispersive x-ray spectroscopy (EDS). The XRD revealed that the as-deposited sample was predominantly amorphous, whereas the annealed samples were polycrystalline with no dominant crystal orientation. The TEM picture as shown in Fig. 7(a) revealed that the crystal grains in the high-temperature-annealed sample changed size and shape partway through the film's thickness, whereas the crystal grains in the low-temperature-annealed samples showed no such change. The EDS result as shown in Fig. 7(b) further showed that in the high-temperature-annealed sample, the oxygen concentration increased sharply partway through the film, whereas the low-temperature-annealed sample showed no such change. These results

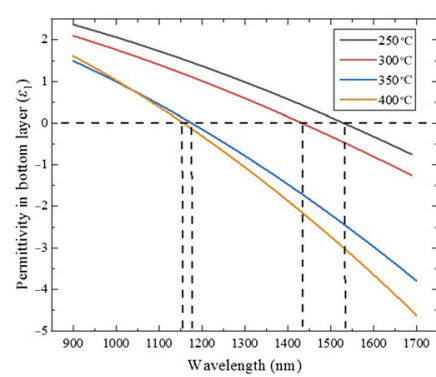

(a)

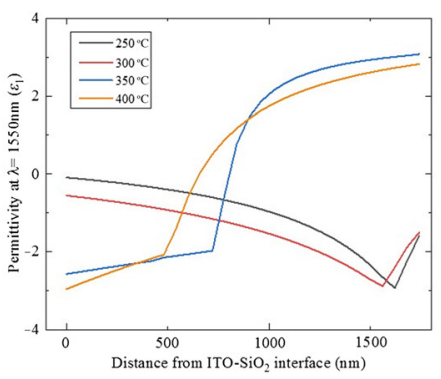

(b)

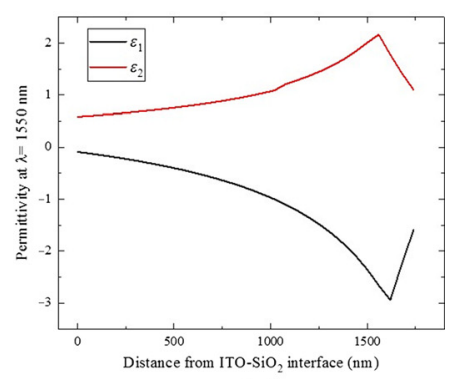

(c)

Fig. 6 (a) ITO metafilms' $\varepsilon_{1}$ as function of wavelength for four different annealing temperatures; (b) $\varepsilon_{1}$ profiles as function of distance from substrate in the $2-\mu \mathrm{m}$-thick ITO film for different annealing conditions; (c) both $\varepsilon_{1}$ and $\varepsilon_{2}$ deep profiles for $250^{\circ} \mathrm{C}$ annealing. 


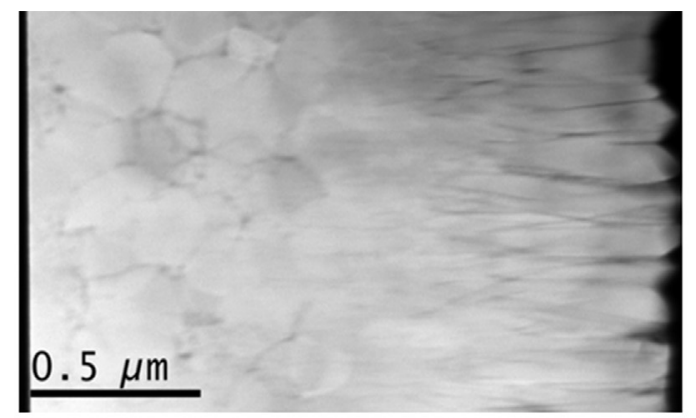

(a)

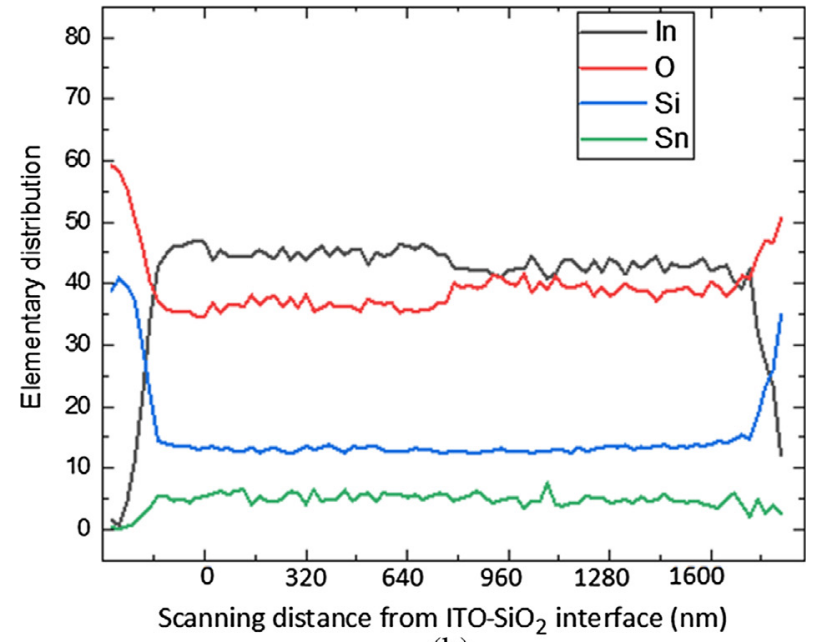

(b)

Fig. 7 (a) Dark field TEM image and (b) EDS plot of the ITO metafilm annealed at $350^{\circ} \mathrm{C}$.

indicate that the crystal grain morphology and oxygen vacancy density may contribute significantly to the permittivity of the ITO film.

We are developing a new fabrication technique to improve the uniformity of the ENZ ITO thick films. The goal is to make both $\varepsilon_{1}$ and $\varepsilon_{2}$ closer to zero through the thickness of the film because higher $\varepsilon_{2}$ will increase the absorption loss for our future device. For this, we deposit the ITO film with high-power impulse magnetron sputtering (HiPIMS), a relatively recent innovation in sputter deposition tools that allows for more control over film growth. Below is the real part and imaginary permittivity behavior of 80 -min annealed sample at $650^{\circ} \mathrm{C}$. The results are shown in Fig. 8. The permittivity depth profile is still not uniform. The real part of the permittivity crosses 0 at $1557 \mathrm{~nm}$, and the imaginary part of the permittivity remains at 0.39 . However, $\varepsilon_{2}$ is closer to zero through the layer thickness compared with our previous ENZ ITO samples.

We are also studying different annealing techniques such as annealing under oxygen gas to control formation of oxygen vacancies in ITO. The detailed results will be discussed in future publications.

\subsection{Design and Modeling of Air-Core, ENZ-ITO Cladding Ring Resonator}

Light-guiding properties of the air-core, ENZ-ITO-cladding ring resonator were numerically modeled using a finite-element-method (FEM) eigenmode solver. The ENZ-ITO resonator consists of a bus waveguide and an optical ring cavity. The bus waveguide and ring cavity guide light in air through a hollow channel embedded in the ENZ-ITO film. The light-guiding mechanism in the hollow waveguide is based on total external reflection (TER) at the air-ITO interface.

In our TER-based waveguide analysis, the fabricated ITO was modeled as a superlattice with 30 sublayers due to the non-uniform distribution of its dielectric constant. The thickness of the individual layer was set to be $60 \mathrm{~nm}$. According to the ellipsometry analysis, the fabricated ITO sample exhibits ENZ properties near the bottom of the film. The region where the absolute value 


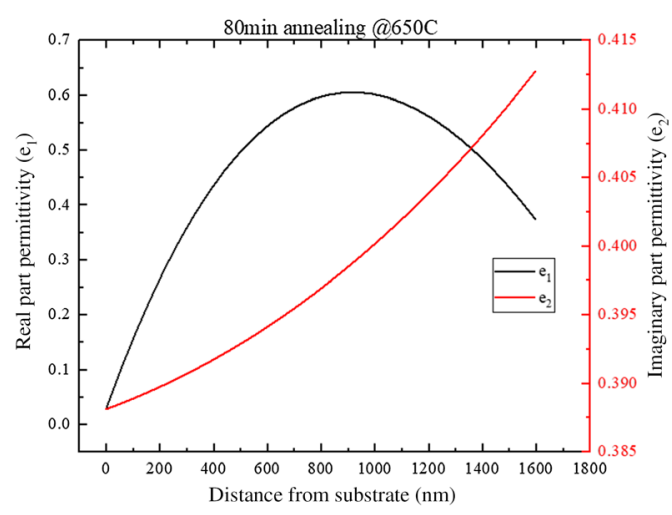

(a)

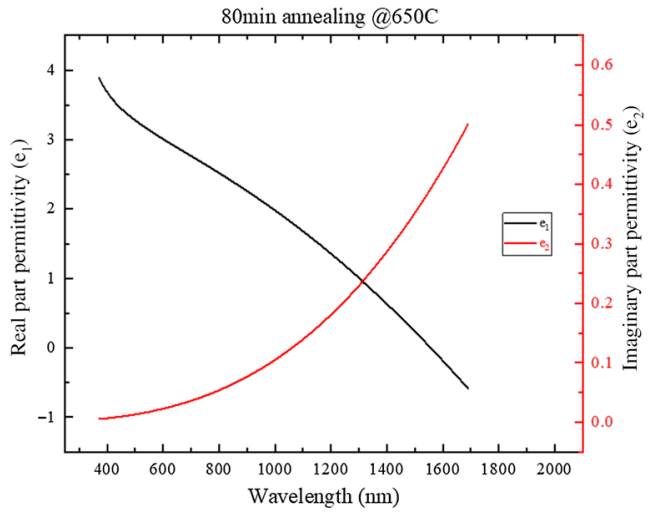

(b)

Fig. 8 The plots of real (black) and imaginary (red) parts of permittivity of ITO metafilms, made by HiPIMS growth and $80 \mathrm{~min}$ of $650^{\circ} \mathrm{C}$ thermal-annealing: (a) as a function of wavelength and (b) as a function of distance from the substrate.

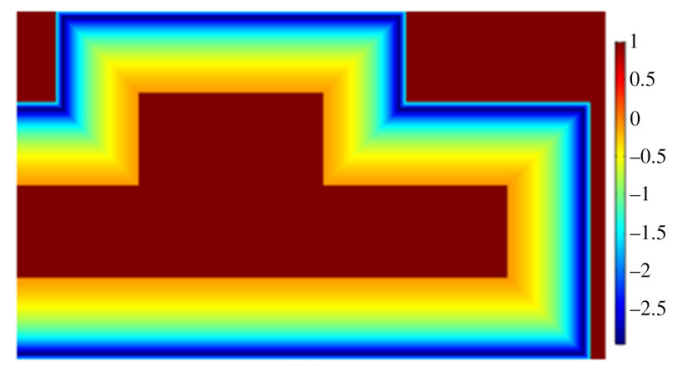

(a)

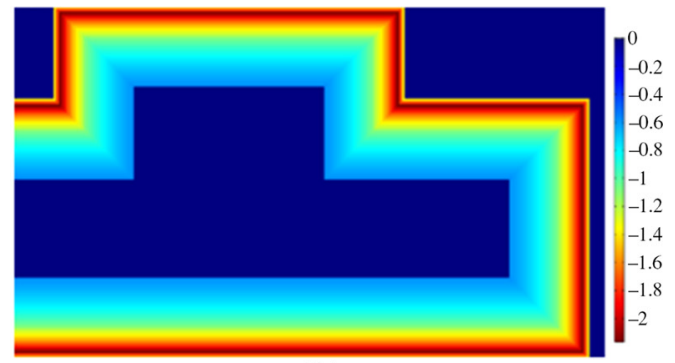

(b)

Fig. 9 (a) The real $\varepsilon_{1}$ and (b) the imaginary $\varepsilon_{2}$ parts of the dielectric constant distribution across the air-core ENZ waveguide.

of the real part, $\varepsilon_{1}$, of the dielectric constant of the ITO is less than 1 was defined as a low-epsilon region. In the air-core waveguide design, this low-epsilon region was used as a cladding layer directly interfacing the air core. Figures 9(a) and 9(b) present the dielectric constant distribution of the air-core ENZ-ITO waveguides. Both real and imaginary parts of the dielectric constant vary gradually along the deposition direction. In this design, a ridge-shape air-core, enclosed by the ITO film, provides the lateral mode confinement.

Figure 10 shows the calculated electric field distribution of the fundamental transverse magnetic mode using a commercial FEM solver, COMSOL Multiphysics ${ }^{\mathrm{TM}}$. The electric field in

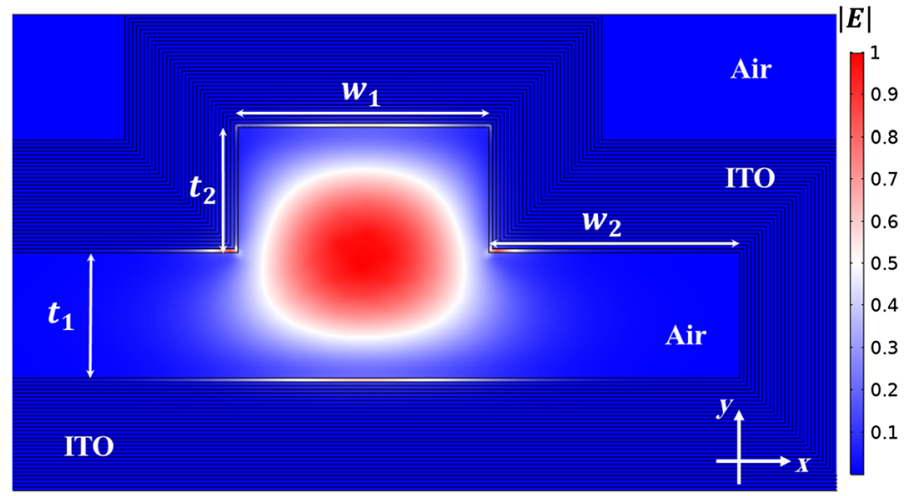

Fig. 10 Calculated electric field profile of the guided mode in the air channel waveguide at $\lambda_{0}=1.55 \mu \mathrm{m}$. 
the air-core waveguide is described by $\mathbf{E}(x, y, z)=\mathbf{E}(x, y) \cdot e^{-j \beta z}$, where $\beta$ is the propagation constant of the guide mode. As shown in the figure, the electric field is tightly confined in the ridge waveguide. Due to the large refractive index contrast between the core (air) and the cladding (ITO), the evanescent tail of the guided mode rapidly decays in the low epsilon region in the ITO film. The simulation parameters used in the mode analysis are $t_{1}=t_{2}=2 \mu \mathrm{m}$, and $w_{1}=w_{2}=4 \mu \mathrm{m}$. The calculated effective refractive index of the fundamental mode is $\beta / k_{0}$ is $0.96641-j 8.3207 \times 10^{-4}$, where $k_{0}\left(=2 \pi / \lambda_{0}\right)$ is the wave vector and $\lambda_{0}$ is the free-space wavelength. The estimated propagation loss is $29.3 \mathrm{~dB} / \mathrm{mm}$.

\subsection{Developing Fabrication Technique for the Air-Core, ENZ-ITO Cladding Ring Resonator}

We have designed several fabrication methods for the air-core, ENZ-ITO-cladding ring resonator. The goal is to use an air cavity as the waveguide core in a ring resonator configuration that is then covered with 1 - to 2- $\mu$ m-thick ENZ ITO material as cladding for the waveguide as shown in Fig. 1. The fabrication process of such device structure involves multiple processing steps. The first processing step is depositing ITO on a $\mathrm{SiO}_{2} / \mathrm{Si}$ substrate. The second step is depositing a sacrificial material layer such as $\mathrm{Si}$ or $\mathrm{Ge}$, and lithographically forming a disk or ring for the resonator. The third step is depositing additional ITO to cover the disk/ring and then annealing it to achieve the desired ENZ property. The final step is etching small windows on top of the disk to expose the sacrificial layer and then remove it using $\mathrm{XeF}_{2}$ vapor.

Due to the nature of ENZ materials, there is some absorption that may increase the propagation loss and reduce the $Q$-factor of the resonator device. Therefore, a major challenge is to reduce the propagation loss for our air-core/ENZ ITO cladding ring-resonator. For that we take two approaches in the fabrication: first, from our experimental data as shown in Figs. 6 and 8, the ENZ ITO layers have lower $\varepsilon_{1}$ and $\varepsilon_{2}$ near the substrate and high $\varepsilon_{2}$ at the top surface, which increases absorption near the surface. Therefore, we developed a fabrication method for a mushroom-shaped sacrificial $\mathrm{Si} / \mathrm{Ge}$ structure, on which we conformally deposit ITO to cover the "mushroom," Figure 11 shows the SEM picture of the mushroom-shaped Si disk and pictures after the ITO is deposited on the sacrificial Si disk. After the annealing process, the ENZ ITO near the interface with the sacrificial $\mathrm{Si} / \mathrm{Ge}$ mushroom has lowest $\varepsilon_{2}$. Therefore, once the sacrificial $\mathrm{Si} / \mathrm{Ge}$ is removed by the vapor etching process, the air-core ring-resonator will be surrounded by low loss ENZ ITO material. Second, using the lithographic process, we created the air-ring resonator with a notched ridge waveguide cross-section shown as Figs. 9 and 10 to reduce interaction between the optical mode and ITO cladding and reduce the amount of light leaking into the ITO.

We are in the process of fabrication and testing such air-ring, ENZ-ITO device structures; further fabrication details and testing results will be reported in future publications.

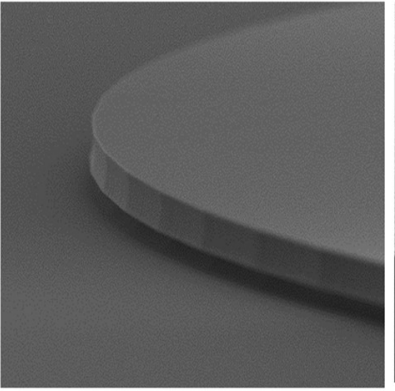

(a)

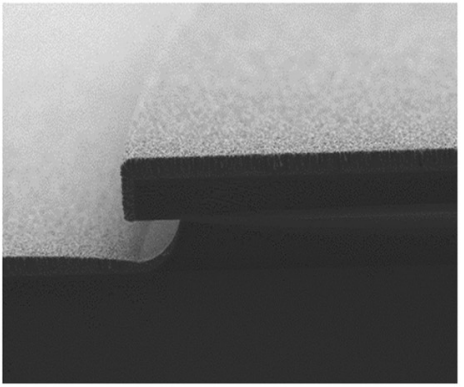

(b)

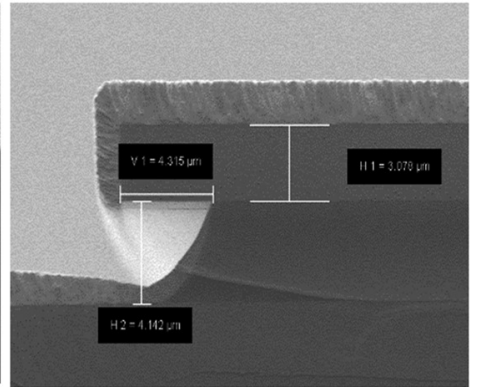

(c)

Fig. 11 (a) An SEM picture of the mushroom-shaped Si disk; (b) and (c) ITO covered Si disk. 


\section{Summary/Conclusion}

We are developing key building block technologies for a long-holdover CSOC. These include a self-locking concept to provide short-term stabilization to a micro-resonator-based OFC. For long-term stabilization, we are developing an environment-insensitive cavity using an ENZ metamaterial. This chip-scale device can be used as oscillator source or local clock in RF and communication systems as well as in 5G networks, and it can potentially be integrated with an optical transmitter and receiver for free-space OTT and ranging to provide positioning, navigation, and timing with low SWaP-C.

\section{Acknowledgments}

We would like thank Professor Kerry Vahala for the invaluable technical discussions and providing high- $Q$ micro-resonators for our study; we thank Professor Curtis Menyuk for providing technical assistance in theoretical soliton study; we thank Professor Rena Huang for helpful discussion for the integrated photonics, and thank Professor Nanshu Lu for the helpful technical discussion in mechanical simulation of the resonator devices; we thank Dr. Jenna Chan and Dr. Jonathan Hoffman for their management and technical support.

\section{References}

1. F. Riehle, "Optical clock networks," Nat. Photonics 11, 25-31 (2017).

2. F. R. Giorgetta et al., "Optical two-way time and frequency transfer over free space," Nat. Photonics 7, 434 (2013).

3. P. Cash et al., "Microsemi chip scale atomic clock (CSAC) technical status, applications, and future plans," in Proc. Eur. Freq. and Time Forum., Turin, pp. 65-71 (2018).

4. S. A. Diddams et al., "An optical clock based on a single trapped ${ }^{199} \mathrm{Hg}^{+}$ion," Science 293(5531), 825-828 (2001).

5. P. Gill, "Optical frequency standards," Metrologia 42(3), S125-S137 (2005).

6. R. Šmid et al., "Precise monitoring of ultra-low expansion Fabry-Perot cavity length by the use of a stabilized optical frequency comb," in Proc. IEEE Int. Frequency Control Symp., pp. 480-484 (2010).

7. P. Brochard et al. "Low-noise transfer of the spectral purity of an optical comb line using a feedforward scheme," J. Eur. Opt. Soc. Rapid Publ. 16, 4 (2020).

8. X. Xiang et al., "Carrier-envelope offset frequency stabilization of a 100 Fs-scale Ti:sapphire mode-locked laser for quantum frequency comb generation," J. Phys. Commun. 2, 055031 (2018).

9. X. Yi et al., "Soliton frequency comb at microwave rates in a high-Q silica microresonator," Optica 2, 1078-1085 (2015).

10. T. Herr et al., "Temporal solitons in optical microresonators," Nat. Photonics 8, 145-152 (2014).

11. X. Yi et al., "Active capture and stabilization of temporal solitons in microresonators," Opt. Lett. 41, 2037-2040 (2016).

12. B. Shen et al., "Integrated turnkey soliton microcombs operated at CMOS frequencies," in Conf. Lasers and Electro-Opt., Paper SF3O.4 (2020).

13. P. Del'Haye et al., "Self-injection locking and phase-locked states in microresonator-based optical frequency combs," Phys. Rev. Lett. 112, 043905 (2014).

14. Z. L. Newman et al., "Architecture for the photonic integration of an optical atomic clock," Optica 6, 680-685 (2019).

15. D. T. Spencer et al., "An optical-frequency synthesizer using integrated photonics," Nature 557, 81-85 (2018).

16. S. Häfner et al., " $8 \times 10^{-17}$ fractional laser frequency instability with a long room-temperature cavity," Opt. Lett. 40, 2112-2115 (2015).

17. F. Kéfélian et al., "Ultralow-frequency-noise stabilization of a laser by locking to an optical fiber-delay line,” Opt. Lett. 34(7), 914-916 (2009). 
18. J. P. Cahill, W. Zhou, and C. R. Menyuk, "Shot noise in self-stabilized optical frequency combs," in Joint Conf. IEEE Int. Freq. Control Symp. and Eur. Freq. and Time Forum, Orlando, Florida, pp. 1-3 (2019).

19. J. P. Cahill, W. Zhou, and C. R. Menyuk, "Self-stabilization of an optical frequency comb using a short-path-length interferometer," Opt. Lett. 42(9), 1680-1683 (2017).

20. S. K. Selvaraja et al., "Highly uniform and low-loss passive silicon photonics devices using a 300 mm CMOS platform," in Proc. Opt. Fiber Commun. Conf., OSA, Washington, DC (2014).

21. M. A. Tran et al., "Ultra-low-loss silicon waveguides for heterogeneously integrated silicon/ III-V photonics," Appl. Sci. 8, 1139 (2018).

22. N. Kobayashi et al., "Silicon photonic hybrid ring-filter external cavity wavelength tunable lasers," J. Lightwave Technol. 33, 1241-1246 (2015).

23. F. Gao et al., "Improvement of sidewall surface roughness in silicon-on-insulator rib waveguides," Appl. Phys. B Lasers Opt. 81, 691-694 (2005).

24. D. J. Blumenthal et al., "Silicon nitride in silicon photonics," Proc. IEEE 106(12), 2209-2231 (2018).

25. M. H. P. Pfeiffer et al., "Ultra-smooth silicon nitride waveguides based on the Damascene reflow process: fabrication and loss origins," Optica 5(7), 884-892 (2018).

26. W. Zhou, "Stabilizing a precision electro-optical oscillator/clock using an environmentinsensitive metamaterial devices," US patent \#:10,756,514B1 (2020).

27. R. Maas et al., "Experimental realization of an epsilon-near-zero metamaterial at visible wavelengths," Nat. Photonics 7, 907-912 (2013).

28. A. Alu and N. Engheta, "Tuning the scattering response of optical nanoantennas with nanocircuit loads," Nat. Photonics 2(5), 307-310 (2008).

29. J. Ni et al., "Property variation in wavelength-thick epsilon near-zero ITO metafilm for near IR photonic devices," Sci. Rep. 10, 713 (2020).

Weimin Zhou is a research physicist and a team leader at the US-Army Research Laboratory (ARL). He is a visionary research leader in the areas of RF-photonics and integrated-photonics, with over 25 patents and 130+ publications. He served as a DARPA's agent on numerous programs and as co-chairs for a number of SPIE and IEEE conferences. He has worked at Bellcore in 1990. He is a fellow of OSA and a senior member of IEEE.

James Cahill received a $\mathrm{PhD}$ in electrical engineering from the University of Maryland, Baltimore County, in 2015 while performing research at the CCDC ARL under the SMART program. He is now a full-time researcher at ARL. His research interests include optical frequency combs, time and frequency metrology, photonic integration, and microwave photonics.

Jimmy H. Ni received his $\mathrm{PhD}$ degree in electrical and computer engineering from the University of Illinois, Urbana-Champaign, in 2017. He joined the ARL as a research engineer in September 2017. He has been working in the area of nanotechnology, metamaterials, and nanophotonics. He has been working on demonstration of metamaterials and nanodevices for RF photonics. He has over 20 technical publications in refereed journals and conference proceedings.

Andrew DeLoach received his PhD in physics from North Carolina State University, Raleigh, North Carolina, in 2013, BS degree in physics from the University of North Carolina at Chapel Hill in 2009. He joined the ARL as a research engineer in 2019.

Sang-Yeon Cho received his PhD from Georgia Institute of Technology, Georgia, in 2003. He was an assistant research professor in the Department of Electrical and Computer Engineering, Duke University, North Carolina. Before joining the ARL in 2019, he worked as an associate professor in the Department of Electrical and Computer Engineering, New Mexico State University, New Mexico. He worked as a visiting faculty researcher at Oak Ridge National Laboratory and Air Force Research Laboratory.

Stephen Anderson is a $\mathrm{PhD}$ candidate at Rensselaer Polytechnic Institute's Department of Electrical, Computer, and Systems Engineering. He is a DoD SMART Fellowship recipient. 
He is conducting his $\mathrm{PhD}$ research at ARL, focusing on integrated RF-photonics, utilizing the slow-light effect and metastructure devices.

Tanvir Mahmood received his $\mathrm{PhD}$ degree in electrical engineering from the University of Maryland, Baltimore County, Maryland, USA. He held technical positions with ClariPhy Communications, Inc., Los Altos, California; Inphi Corporation, Santa Clara, California; and was affiliated with Laboratory for Physical Sciences, College Park, Maryland. He is currently working as a postdoctoral research fellow at US ARL. His research interests include nonlinear optics, microwave photonics, optical communication systems, and integrated optics.

Patrick Sykes is a graduate student in the Department of Electrical and Computer Engineering at University of Maryland, Baltimore County. He is a DoD SMART Fellowship recipient and conducting an experimental research on optical time transfer at ARL.

Wendy L. Sarney is a physical scientist at the CCDC Army Research Lab. She received her $\mathrm{PhD}$ in materials science from the University of Maryland, College Park, in 2000, and her BS degree in physics from Binghamton University in 2015. Her principal research techniques have included transmission electron microscopy, molecular beam epitaxy, and x-ray diffraction. She has over 140 technical publications in refereed journals and conference proceedings.

Asher C. Leff received his BS and MS degrees in materials science and engineering from Drexel University in 2013 before completing his $\mathrm{PhD}$ in materials science and engineering at Drexel in 2017. During his PhD, he developed new TEM techniques including pioneering work on directelectron-detection EELS and dislocation density mapping. Since 2017, he has worked as the TEM scientist for ARL-SEDD. His primary research interests are solid-state thermal energy storage and 3D electrical energy storage devices. 